\title{
ReaR
}

ISNN 19894090

Revista electrónica de AnestesiaR

Febrero 2022

CASOS CLÍNICOS

\section{Consideraciones perioperatorias del síndrome de Noonan en el adulto.}

Martínez Saniger A, Guerrero Domínguez R, Rubio Romero R, Jiménez I.

Hospital Universitarios Virgen del Rocío, Sevilla, España.

\section{Resumen}

El síndrome de Noonan (SN) es una enfermedad monogénica con patrón de herencia autosómico dominante con una incidencia de uno de 1:1500-2500. Este síndrome es de particular interés para el anestesiólogo por ser considerado probable vía aérea difícil, presentar talla baja, dismorfia facial, alteraciones en la hemostasia, musculoesqueléticas y cardiológicas, entre otras, cuya evolución condicionan la esperanza de vida alcanzando edades más avanzadas en la actualidad gracias al control de las mismas. Por consiguiente requieren intervenciones en la edad adulta donde no hay tantos casos reportados como en la infancia. Presentamos un caso de un paciente de 53 años con SN programado para resección de neurinoma cervical durante el cual destacó un sangrado aumentado durante la cirugía no esperado. Nuestro objetivo es establecer un plan anestésico específico tras exponer las características de la enfermedad para minimizar las complicaciones perioperatorias.

\section{Introducción}

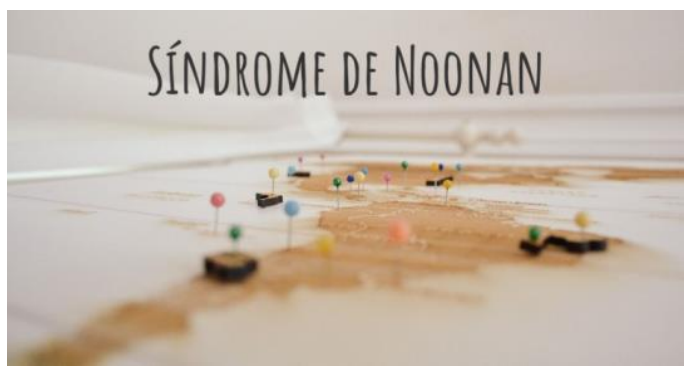

El síndrome de Noonan (SN) es una enfermedad monogénica con patrón de herencia autosómico dominante con una incidencia de uno de 1:1500-2500. Este síndrome es de particular interés para el anestesiólogo por ser considerado probable vía aérea difícil, presentar talla baja, dismorfia facial, alteraciones en la hemostasia, musculoesqueléticas y cardiológicas, entre otras, cuya evolución condicionan la esperanza de vida alcanzando edades más avanzadas en la actualidad gracias al control de las mismas . Por consiguiente requieren intervenciones en la edad adulta donde no hay tantos casos reportados como en la infancia . Presentamos un caso de un paciente de 53 años con SN programado para resección de neurinoma cervical durante el cual destacó un sangrado aumentado durante la cirugía no esperado. Nuestro objetivo es establecer un plan anestésico específico tras exponer las características de la enfermedad para minimizar las complicaciones perioperatorias.

Alrededor del $60 \%$ de los casos de $\mathrm{SN}$ son causados por mutaciones de tipo missense en el gen PTPN1, las cuales provocan una actividad aumentada de la proteína que codifica el gen, una tirosinfosfatasa citoplasmática SHP2. Esta proteína interviene en la vía de señalización intracelular RAS MAPK, responsable de controlar el crecimiento, diferenciación, migración y apoptosis celular de factores de crecimiento y hormonas en varias fases del desarrollo físico y mental (2). 
Clínicamente se caracteriza por ser la segunda causa congénita de talla baja, tras el síndrome de Down.

Estos pacientes presentan fascies en forma de triángulo invertido, hipertelorismo, desviación ocular antimongoloide, ptosis, epicantus, orejas bajas y rotadas con hélix grueso(3).

Desde el punto de vista cardiológico las manifestaciones clínicas más representativas engloban la cardiopatía estructural (50-80\%), siendo la estenosis pulmonar con displasia valvular $(20-50 \%$ de los casos) y la miocardiopatía hipertrófica $(\mathrm{MCH})(20-$ $30 \%)$ los hallazgos más frecuentes $(1,4)$ pudiendo causar esta última obstrucción del tracto de salida del ventrículo izquierdo. Otras afecciones cardiológicas menos comunes abarcan los defectos en el septo, estenosis de ramas pulmonares, tetralogía de Fallot y coartación aórtica(2).

Presentan alteraciones esqueléticas, como cifoescoliosis y pectum carinatum o excavatum, malformaciones genitales y renales, así como mayor riesgo de desarrollo de tumores sólidos, como lesiones de células gigantes, rabdomiosarcomas embrionarios, neuroblastomas, tumores genitales, cutáneos y hematológicos.

En ocasiones existe disminución del desarrollo psicomotor, como consecuencia de la hipotonía e hiperlaxitud articular, y bajo coeficiente intelectual(15-35\%)(2). Pueden presentar falta de cooperación previa a la inducción anestésica, siendo necesaria una mayor premedicación, aunque la mayoría de los pacientes resultan colaboradores(5).

Se ha descrito inestabilidad atlantoaxoidea que, junto con un cuello grueso $y$ ancho, la presencia de maloclusión dental y las alteraciones en el paladar, predisponen a una probable vía aérea difícil. Por todo ello se recomienda intubación con videolaringoscopio o fibrobroncoscopio (FB) despierto $(4,6)$.

Hay descritas diversas anomalías de la coagulación, que afectan tanto a la hemostasia primaria, con disfunción plaquetaria, como a la secundaria, casi en el $60 \%$ de los pacientes. En su mayoría consisten en una combinación leve de deficiencias de varios factores de coagulación (V, VII, VIII, IX, X, XI) que causan un tiempo de protrombina (TP) y/o tiempo de tromboplastina parcial activada (TTPa) alargados, o defectos del factor de Von Willebrand. No obstante, en muchas ocasiones estos defectos no se detectan con las pruebas de laboratorio habituales donde los tiempos resultan normales(7).

Presentamos el caso de un paciente adulto con SN sometido a una intervención neuroquirúrgica.

\section{Caso Clínico}

Varón de 53 años con SN y lesión cervical C5 sugestiva de neurinoma, propuesto para cirugía de exéresis.

Como antecedentes personales destacan alergia a penicilinas e hipotiroidismo primario.

En la exploración física encontramos talla baja $(149 \mathrm{~cm})$, implantación baja de pabellones auriculares, pterigium colli, cúbito valgo hendido, pectum excavatum, cifoescoliosis y acromicria.

Valorado en cardiología por un soplo asintomático que en la última ecocardiografía se informó como insuficiencias mitral, aórtica y pulmonar ligeras, disfunción diastólica tipo I, ventrículo izquierdo ligeramente dilatado con rodete septal hipertrófico y 
fracción de eyección ventricular conservada. Como cirugías previas había precisado la resección de un tumor de células gigantes en cavidad oral, orquidopexia y hernioplastia inguinal realizadas en otro centro sin conocer el desarrollo de las cirugías ni la técnica anestésica.

En la exploración de la vía aérea se observa la ausencia de dentición, con Mallampati I, apertura bucal normal, ausencia de limitación a la extensión cervical, micrognatia y lipodistrofia facial.

El electrocardiograma mostró un ritmo sinusal a $73 \mathrm{lpm}$ y hemibloqueo anterior izquierdo con hipertrofia ventricular izquierda.

El hemograma presentó una hemoglobina de 10,3 g/dl, una anemia microcítica e hipocrómica asociada a sangrado hemorroidal. Se había tratado con hierro oral, aumentando la cifra de hemoglobina hasta $12,9 \mathrm{~g} / \mathrm{dl}$ el día previo a la cirugía. El estudio de coagulación fue normal. Se solicitó tipaje sanguíneo con escrutinio de anticuerpos irregulares y se pautó premedicación con $6 \mathrm{mg}$ de midazolam oral.

Se monitorizó intraoperatoriamente con electrocardiograma continuo DII y V5, pulsioxímetro y presión arterial no invasiva. Se administró profilaxis antibiótica con $1 \mathrm{~g}$ de vancomicina. La inducción anestésica se realizó con 2 $\mathrm{mcg} / \mathrm{kg}$ de fentanilo, $2 \mathrm{mg} / \mathrm{kg}$ de propofol y $0,6 \mathrm{mg} / \mathrm{kg}$ de rocuronio previa carga de $500 \mathrm{ml}$ de Isofundín. Se añadió dexametasona $8 \mathrm{mg}$ para atenuar el edema medular por la manipulación de la cirugía. Para la ventilación manual se precisaron dos anestesiólogos por fuga perimascarilla a pesar de usar cánula orofaríngea. La intubación orotraqueal se realizó con videolaringoscopio GlideScope sin incidencias, teniendo disponible el FB. Los parámetros respiratorios se ajustaron a un VT de $6 \mathrm{ml} / \mathrm{kg}$ sin dificultad en la ventilación a pesar del patrón restrictivo ventilatorio esperable. El mantenimiento anestésico se realizó con propofol en "Target- controlled infusion system" (TCI) siguiendo el modelo de Marsh, con una concentración plasmática objetivo que osciló entre $2-3 \mathrm{mcg} / \mathrm{ml}$ para mantener un índice biespectral (BIS) entre 40-60, $\mathrm{y}$ remifentanilo en perfusión $0.05-0.15$ $\mathrm{mcg} / \mathrm{kg} / \mathrm{min}$ manteniendo tensión arterial media (TAM) entre 65-70 $\mathrm{mmHg}$, garantizando así una adecuada presión de perfusión sanguínea medular. Se colocó al paciente en decúbito prono discurriendo la cirugía sin incidencias hemodinámicas, aunque sí con cierta tendencia al sangrado que dificultaba la visualización por el equipo de neurocirujanos a través del microscopio quirúrgico.

No obstante, el sangrado intraoperatorio no superó los $300 \mathrm{ml}$.

Se administró $1 \mathrm{~g}$ de paracetamol y 100 mcg de fentanilo iv como rescate analgésico y se revirtió el bloqueo neuromuscular con $2 \mathrm{mg} / \mathrm{kg}$ de sugammadex procediéndose a la extubación sin incidencias.

Se trasladó a la unidad de recuperación postanestésica para cuidados postoperatorios permaneciendo 5 horas hasta su traslado a planta con estabilidad hemodinámica y hemoglobina postquirúrgica de 10,3 $\mathrm{g} / \mathrm{dl}$.

\section{Discusión}

McLure y Yentis(8) describen el uso de anestesia general para la realización de una cesárea en una paciente con SN. Los autores decidieron asegurar la vía aérea mediante una intubación con FB dadas las múltiples anomalías faciales 
como micrognatia y limitación de la apertura bucal.

En un artículo de Chase et al(1) sobre cesárea bajo anestesia general se describe el empleo exitoso de videolaringoscopio ya que mejora la exposición laríngea y facilita el primer intento de intubación endotraqueal difícil. Esta fue la técnica usada en nuestro caso ya que la apertura bucal era buena y no había constancia de dificultades especiales en la intubación en cirugías previas según la entrevista con el paciente. No obstante, es recomendable disponer de FB dentro del equipamiento de vía aérea por si fuera necesario(1).

Aunque no es el caso que exponemos, mencionar que en los pacientes subsidiarios de anestesia espinal hay que tener en cuenta que las anomalías vertebrales pueden suponer dificultades técnicas(1), no obstante, hay descritos casos de cesáreas electivas con anestesia epidural(5) sobre todo si se asocia a cardiopatía hipertrófica.

Resulta fundamental la prevención y tratamiento de los episodios de taquicardia en relación a la estimulación simpática generada por el estrés quirúrgico $(4,6)$, así como evitar episodios de hipotensión arterial e hipoxemia(4).

El fentanilo y remifentanilo han sido empleados como analgésicos de elección ya que conllevan pocos efectos sobre la contractilidad miocárdica(4). E1 propofol disminuye las resistencias vasculares sistémicas (RVS) y el retorno venoso, mientras que la ketamina causa hipertensión arterial y taquicardia. Ambos deberían evitarse durante la inducción de la anestesia en aquellos pacientes con cardiopatía inestable(6). El etomidato por sus propiedades farmacológicas resulta cardioestable e indicado en los casos con $\mathrm{MCH}$ obstructiva(9). En nuestro caso optamos por una inducción con propofol por tener preservada la contractibilidad cardíaca y posterior mantenimiento anestésico con el mismo fármaco. Se ha considerado que debe evitarse el uso de halogenados ya que, aunque la hipertermia maligna es rara, podría darse en los pacientes con $\mathrm{SN}(10)$. No obstante, en casos reportados más recientes, no se menciona esta asociación usando halogenados para el mantenimiento anestésico(1-4,6) por su buena tolerancia cardiológica.

En los pacientes con $\mathrm{MCH}$ se recomienda optimizar la fluidoterapia garantizando una adecuada normovolemia, evitando episodios de hipotensión arterial durante la inducción anestésica(9).

La depresión miocárdica dependiente de los agentes anestésicos halogenados resulta útil para generar una respuesta inotrópica negativa leve y mantener un gradiente de presión ventricular izquierdo adecuado(4). El isoflurano y el desflurano cuando se administran en altas concentraciones aumentan la frecuencia cardiaca y la presión arterial con lo cual habría que extremar las precauciones en su uso.

El efecto vasodilatador cerebral es menor con el sevoflurano, siendo el anestésico halogenado de elección durante el mantenimiento anestésico, ya que causa una disminución moderada en las RVS y la presión arterial, con una acción mínima en el flujo sanguíneo cerebral(6). No obstante, en este caso no pudo ser empleado por su conocido efecto en la monitorización neurofisiológica intraoperatoria. Puesto que la cardiopatía conocida fue considerada estable, se empleó propofol tanto en la inducción como el mantenimiento anestésico. 
En estadios más avanzados de $\mathrm{MCH}$, complicaciones como la insuficiencia cardíaca congestiva, la isquemia miocárdica, la hipotensión sistémica y las arritmias supraventriculares $\mathbf{O}$ ventriculares podrían precipitarse(6), por lo que debe haber vigilancia estrecha y tratamiento precoz.

En nuestro caso, el estudio de coagulación rutinario resultó normal, aunque el cirujano refería cierta tendencia al sangrado que dificultaba la resección tumoral. Los pacientes con SN presentan alteraciones de la hemostasia. No hay bibliografía aún sobre el uso de ROTEM/TEG de manera preoperatoria para su detección pero sí recomendación de revisión por un hematólogo previa cirugía(7). En nuestro caso el sangrado dificultaba la resección tumoral mediante microcirugía.

En nuestro paciente fue preciso optimizar el nivel de hemoglobina preoperatorio con hierro oral, $\mathrm{y}$ adoptamos medidas intraoperatorias para reducir el sangrado con una hemostasia quirúrgica exhaustiva $\mathrm{y}$ hemostáticos locales, correcto posicionamiento del paciente y control de la TAM, y se adoptó una estrategia restrictiva transfusional perioperatoria en función de la tolerancia a la anemia, que permitió no transfundir al alta hospitalaria.

Debido a que el diagnóstico es clínico la enfermedad puede estar infradiagnosticada(6), por lo que debe establecerse un adecuado diagnóstico diferencial con otras entidades clínicamente muy similares y con alteraciones también en la vía RASMAPK como el síndrome cardiofaciocutáneo, síndrome de Costello, Neurofibromatosis tipo 1 y SN con lentiginosis múltiple, entre otros, todos ellos englobados con el término rasopatías.
En conclusión, muchos de los pacientes con SN no alcanzan la edad adulta, por lo que resulta infrecuente que se sometan a cirugías en esta edad.

Es de vital importancia el adecuado control de la vía aérea, así como de las dificultades asociadas a las anomalías anatómicas y fisiopatológicas con el fin de garantizar un adecuado manejo anestésico disminuyendo la morbimortalidad en el escenario quirúrgico(4). Todos los pacientes con SN deben ser informados de que tienen mayor riesgo de sangrado durante procedimientos quirúrgicos. A pesar de que el TP y TTPa no revelan defectos de coagulación, todavía existe un riesgo potencial de hemorragia, por tanto, podrían beneficiarse de ser derivados a un hematólogo para investigaciones preoperatorias y para el manejo perioperatorio adaptado(7). Artoni et al(11) concluye que se debería hacer un screening de factores de coagulación y pruebas de función plaquetarias y tratar preoperatoriamente si existiera alguna anomalía. En nuestro caso, no se realizó un estudio hematológico exhaustivo que quizá podría haber mejorado el campo quirúrgico si se hubiese detectado alguna alteración.

Debería emplearse una monitorización adecuada con el fin de anticipar eventos adversos relacionados con la técnica anestésica, fundamentalmente en aquellos casos con patología cardiaca asociada.

\section{Bibliografía}

1. Chase CJ, Holak EJ, Pagel PS. Anesthetic implicactions of emergent Cesarean section in a parturient with Noonan syndrome and bacterial endocarditis. J Clin Anesth 2013; 25:403-406. DOI: 10.1016 / j.jclinane.2012.11.016 ( $\underline{\text { HTML) }}$

2. Ballesta-Martínez MJ, Guillén-Navarro E. Síndrome de Noonan. AEP Protocolos 2010: 56-63. ISSN 2171-8172. (HTML) 
3. Asahi Y, Fujii R, Usue N, Kagamiuchi J, Omichi S, Kotani J. Repeated general anesthesia in a patient with Noonan Syndrome. Anesth Prog 2015; 62.71-73. DOI: 10.2344 / 00033006-62.1.71. ( PubMed)

4. Singh Bajwa SJ, Gupta S, Kaur J, Panda A, Bajwa SK, Singh A, Parmar SS et al. Anesthetic consideratios and difficult airway management in a case of Noonan syndrome. Saudi J Anaesth 2011; 5(3): 345-347. DOI: 10.4103 / 1658354X.84121 (PubMed)

5. McBain J, Lemire EG, Campbell DC. Epidural labour analgesia in a parturient with Noonan syndrome: a case report. Can J Anesth 2006; 53 (3): 274-278. DOI: 10.1007 / BF03022215 (ㄴML)

6. Samra T, Banerjee N. Anesthesia for emergency ventriculo-peritoneal shunt in an adolescent with Noonan's syndrome. Indian J Aaesth 2014; 58(4): 452-455. DOI: 10.4103 / 0019-5049.138991 (MC)

7. Morice A, Harroche A, Cairet P, Khonsari RH. Preoperative Detailed Coagulation Tests Are Required in Patients with Noonan Syndrome. J Oral Maxillofac Surg. 2018 Jul;76(7):1553-1558.

doi: 10.1016/j.joms.2017.12.012. Epub 2017 Dec 29 (PubMed)
8. McLure HA, Yentis SM. General anaesthesia for caesarean section in a parturient with Noonan's syndrome. Br J Anaesth 1996;77:6658. DOI: 10.1093 / bja / 77.5.665 (ㄴML)

9. Aggarwal V, Malik V, Malthotra Kapoor P, Kiran U. Noonan syndrome: an anesthesiologist's perspective. Annals of Cardiac Anaesthesia 2011; 14(3): 214-217. DOI: 10.4103 / 0971-9784.84024 (HTML)

10.Lee CK, Chang BS, Hong YM, Yang SW, Lee CS, Seo JB. Spinal deformities in Noonan syndrome: a clinical review of sixty cases. J Bone Joint Surg. 2001; 83:1495-1502 (․ubMed)

11. Artoni A, Selicorni A, Passamonti SM, Lecchi A, Bucciarelli P, Cerutti M, Cianci P, Gianniello F, Martinelli I: Hemostatic abnormalities in Noonan syndrome. Pediatrics 133: $\quad$ e1299, 2014. DOI:https://doi.org/10.1542/peds.2013-3251 (PubMed)

Correspondencia al autor

Ana Martinez Saniger

ana.martinezsa@gmail.com

FEA Anestesiología y Reanimación

Hospital Universitario Virgen del Rocio, Sevilla, España.

Aceptado para el blog en abril de 2021 